\title{
Reactivation of TAp73 tumor suppressor by protoporphyrin IX, a metabolite of aminolevulinic acid, induces apoptosis in TP53-deficient cancer cells
}

\author{
Alicja Sznarkowska', Anna Kostecka1', Anna Kawiak', Pilar Acedo², Mattia Lion ${ }^{3,4}$, Alberto Inga ${ }^{3}$ \\ and Joanna Zawacka-Pankau ${ }^{2 *}$ (1)
}

\begin{abstract}
Background: The p73 protein is a tumor suppressor that shares structural and functional similarity with p53. p73 is expressed in two major isoforms; the TA isoform that interacts with p53 pathway, thus acting as tumor suppressor and the N-terminal truncated $\Delta \mathrm{N}$ isoform that inhibits TAp73 and p53 and thus, acts as an oncogene.

Results: By employing a drug repurposing approach, we found that protoporphyrin IX (PpIX), a metabolite of aminolevulinic acid applied in photodynamic therapy of cancer, stabilizes TAp73 and activates TAp73-dependent apoptosis in cancer cells lacking p53. The mechanism of TAp73 activation is via disruption of TAp73/MDM2 and TAp73/MDMX interactions and inhibition of TAp73 degradation by ubiquitin ligase Itch. Finally, PpIX showed potent antitumor effect and inhibited the growth of xenograft human tumors in mice.
\end{abstract}

Conclusion: Our findings may in future contribute to the successful repurposing of PpIX into clinical practice.

Keywords: TAp73, Protoporphyrin IX, Apoptosis, Yeast-based assay, MDM2, MDMX, Itch

\section{Background}

Drug repurposing brings hope for the improved treatment of cancer patients due to the financial toxicity of current cancer care that impacts both, patient care and healthcare system worldwide [1].

p73 and p63 are structural and functional homologs of the p53 tumor suppressor protein. All p53 family proteins share the ability to activate p53 target genes involved in apoptosis and cell cycle control under stress conditions.

The TP73 gene structure is complex due to the existence of two alternative promoters (P1 and P2) (Fig. 1a). The P1 promoter drives the expression of transcriptionally active TAp73 isoforms, acting as tumor suppressors, while expression from $\mathrm{P} 2$ promoter generates $\mathrm{N}$-terminal

\footnotetext{
*Correspondence: joannazawackapankau1@gmail.com

${ }^{2}$ Department of Microbiology, Tumor and Cell Biology, Karolinska Institutet, Biomedicum, Solnavägen 9, 17165 Stockholm, Sweden Full list of author information is available at the end of the article
}

truncated $\triangle \mathrm{Np} 73$ isoforms, acting as dominant negative towards TA isoforms and p53 proteins, and thus known as oncogenes [2]. It has been shown that TA and $\Delta \mathrm{N}$ isoforms possess distinct, often opposing functions in healthy and cancerous tissues [3]. Next, it has been demonstrated that the outcome of chemotherapy depends on the TA and $\triangle \mathrm{N}$ isoforms ratio [4].

Unlike TP53, the gene encoding TP73 is rarely mutated in cancers and the functional isoforms are expressed in the majority of human tumors [5]. TAp73 is found inactivated in cancers by binding to oncogenic $\triangle \mathrm{Np} 73$, MDM2 and MDMX proteins or by degradation by the ubiquitin ligase Itch [6, 7]. Previous studies point at the possibility of exploiting TAp73 as a tumor suppressor for improved cancer therapy. For example it has been demonstrated that p73 via activation of c-Jun N-terminal Kinase (JNK) drives the sensitivity to cisplatin in ovarian cancer cells independent on the p53 status [8]. Next, Nutlin3, a selective inhibitor of MDM2, stabilizes TAp73 and induces 
(See figure on next page.)

Fig. 1 PpIX inhibits proliferation of cancer cells lacking p53. a Schematic representation of the splice variants in TP73 gene (upper panel) and domain structure of p73 (lower panel). TA transactivation domain, DBD DNA binding domain, OD oligomerization domain, CT C terminus. b PpIX induces dose-dependent growth inhibition in a long-term proliferation assay. $\mathbf{c}$ Ectopic expression of TAp73a sensitizes cells to PpIX after $24 \mathrm{~h}$ as demonstrated by WST-1 proliferation assay. Inserted blot represents the level of expression of TAp73a. Please note that the blot has been cropped. Dotted line represents where the blot has been cut. The uncropped full length version is presented in Additional file 3: Figure S3a. d TAp73a overexpression sensitizes H1299 to PpIX-induced inhibition of proliferation. e, f PpIX does not induce DNA damage in cancer cells at the effective therapeutic concentrations. $\mathbf{g} 2.5 \mathrm{\mu g} / \mathrm{ml}$ PpIX induces PARP-1 cleavage in HCT 116 p53-/- but not in non-transformed human diploid fibroblasts. Dotted line represents where the blot has been cut. The uncropped blot is presented in Additional file 3: Figure S3b

TAp73-mediated apoptosis [9]. It has also been demonstrated that simultaneous induction of proteotoxic and oxidative stress leads to JNK-induced phosphorylation of TAp73 and TAp73-mediated cell death in p53-null tumors [10].

Small molecule protoporphyrin IX (PpIX), is a natural metabolite of $\delta$-aminolevulinic acid, a pro-drug applied in clinics in photodynamic therapy of cancer (PDT) [11]. It has also been tested as a radiosensitizer in mouse mammary tumours [12]. PpIX induces HeLa cells' apoptosis per se, without light excitation [13], stabilizes and activates wild-type p53 in human colon carcinoma cells [14] and binds to p73 [15].

Here, we have found that PpIX activates TAp73 in cancer cells lacking TP53 gene. We demonstrated that PpIXactivated TAp73 compensates for p53 loss in cancer cells and induces apoptosis. The mechanism of transcriptional activation of TAp73 by PpIX is via inhibition of TAp73/ MDM2 and TAp73/MDMX interactions. TAp73 protein stabilization is achieved by disrupting TAp73/Itch complex by PpIX. Furthermore, PpIX inhibits tumors growth in xenograft experiments.

\section{Results}

\section{Protoporphyrin IX inhibits proliferation and induces} apoptosis in TP53-null cancer cells

It has been demonstrated that PpIX per se, activates wildtype $\mathrm{p} 53$ by disrupting p53/MDM2 complex and induces p53-dependent and independent apoptosis in human colon cancer cells [14]. Since PpIX binds to p73 [15] we reasoned that $\mathrm{p} 73$ might play role in mediating cell death in p53-null cancer cells upon PpIX treatment. Here, in long-term proliferation assay, we showed that PpIX inhibits growth of several TP53-null cancer cell lines in a dose-dependent way (Fig. 1b). Interestingly, overexpression of TAp73 $\alpha$ sensitized cancer cells to PpIX as shown in short- and long-term proliferation assays (Fig. 1c, d). Thus, accumulated TAp73 contributed to cancer cells' susceptibility to PpIX.

Next, we assessed the genotoxicity of PpIX. The comet assay showed that PpIX did not induce DNA damage in human colon cancer cells (Fig. 1e, f) at effective concentrations against cancer cells under in vitro conditions. Importantly, western blotting demonstrated PARP-1 cleavage, indicating active apoptosis, only in cancer cells but not in normal human diploid fibroblasts (NHDF) after protoporphyrin IX treatment (Fig. 1g). A slight reduction in total PARP-1 was detected in NHDF after PpIX and cisplatin (CDDP) but only CDDP induced PARP-1 cleavage in these cells. This implies that PpIX is non-genotoxic and does not affect normal cells at concentrations tested in comparison to cisplatin.

\section{Protoporphyrin IX induces TAp73 and its apoptotic target genes in TP53-null cancer cells}

To assess the potential of PpIX against other TP53-null human cancer cells than HCT 116 p53-/-, we employed human lung adenocarcinoma cells (H1299) and human osteosarcoma cells (Saos2). The caspase assay showed potent induction of apoptosis by PpIX after $6 \mathrm{~h}$ in all three cell lines tested (Fig. 2a and Additional file 1: Figure S1a). Induction of active caspases by PpIX correlated with the accumulation of cleaved PARP-1 in H1299 (Fig. 2b).

TAp73 and p53 recognize the same target genes involved in the apoptotic response. We found that PpIX at the concentrations from 1 to $5 \mu \mathrm{g} / \mathrm{ml}$ induces accumulation of TAp73 with the concomitant upregulation of its apoptotic targets Noxa and PUMA on both mRNA and protein levels (Fig. 2c-e). At the same time we observed the downregulation of the oncogenic $\Delta \mathrm{N}$ isoform of p73 (Fig. 2f). The induction of TAp73 and its pro-apoptotic targets was also detected for Nutlin3 and cisplatin (Fig. 2d, e, respectively). Taken together, PpIX stabilizes TAp73 and induces apoptosis in cancer cells lacking TP53 in a dose- and time-dependent manner.

\section{PplX-activated TAp73 compensates for $\mathrm{p} 53$ loss in inducing apoptosis in TP53-null cancer cells}

To determine if TAp73 is critical for PpIX-induced cancer cells' death, we depleted TAp73 using isoform specific siRNA. PpIX had no effect on the mRNA levels of TAp73 upon the knockdown as demonstrated by real-time PCR (Fig. 3a). 


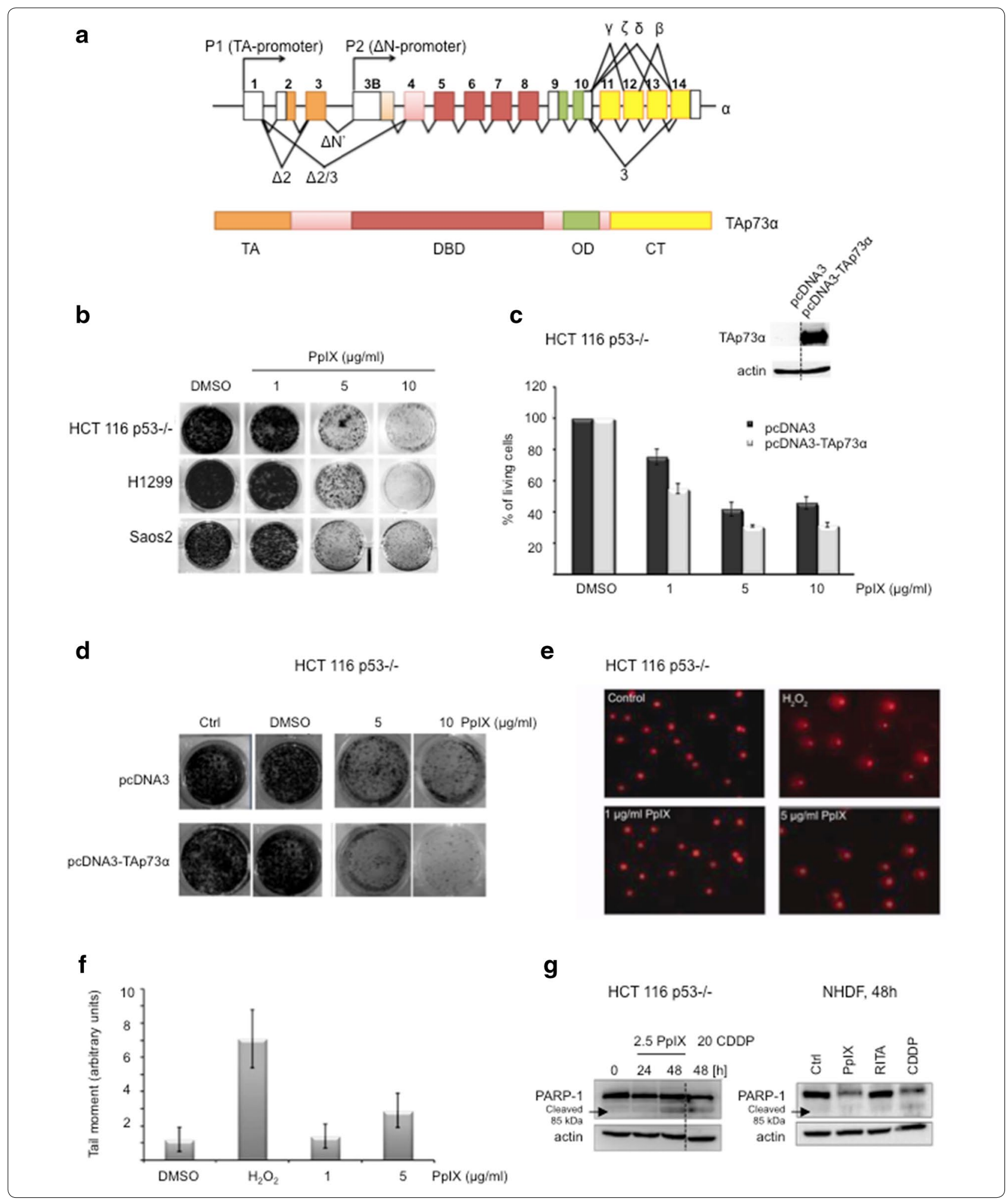

The knockdown of TAp73 led to resistance to PpIX as shown in the long-term proliferation assay (Fig. $3 \mathrm{~b}$ ) and caspase assay (Additional file 2: Figure S2a). Next, we showed that silencing of TAp73 abolished PpIX-mediated induction of Noxa and Puma as well as PARP-1 cleavage (Fig. 3c, d and Additional file 2: Figure S2b). 


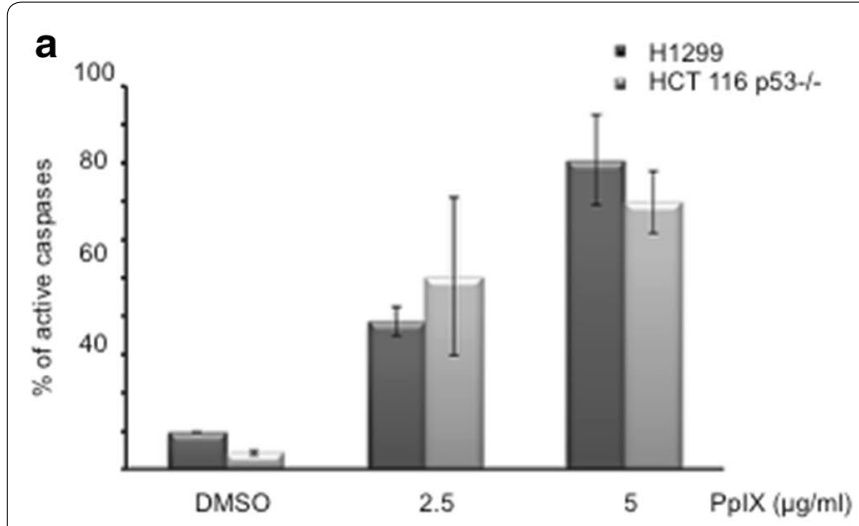

c

H1299

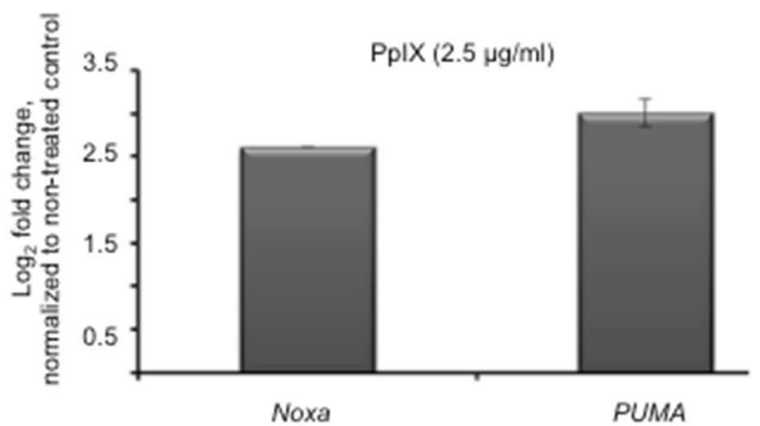

e

HCT 116 p53-/-

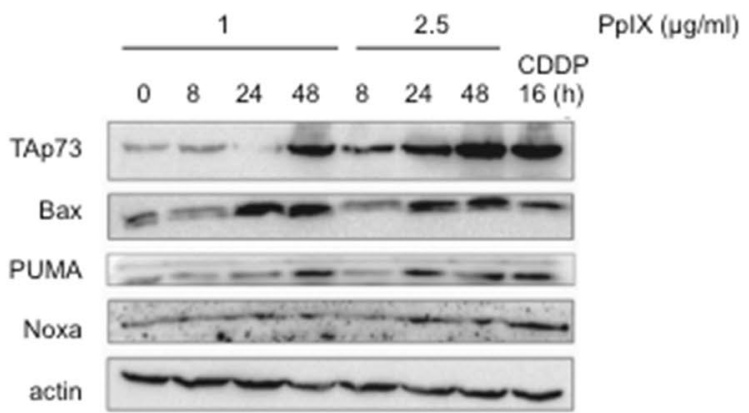

b

$\mathrm{H} 1299$

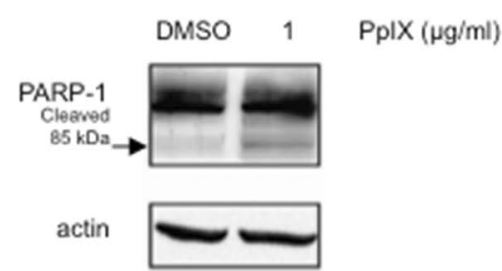

d

H1299

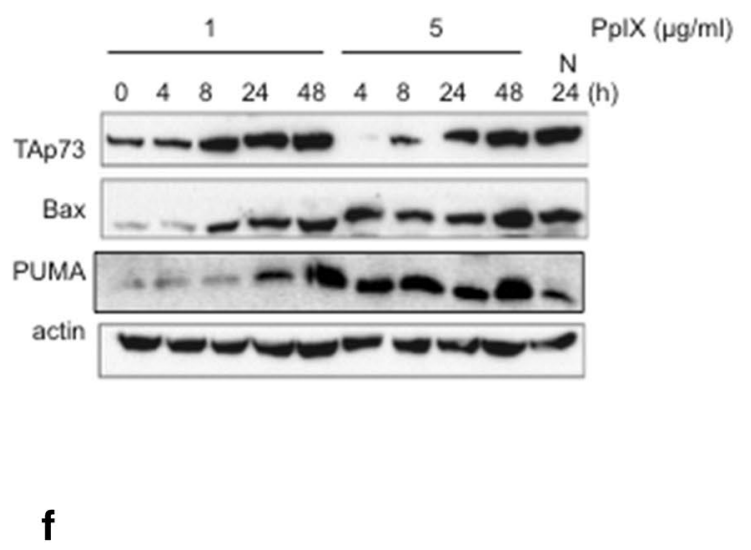

HCT 116 p53-/-

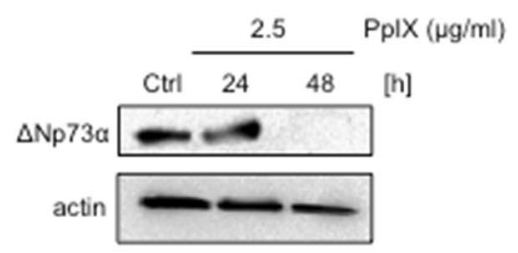

Fig. 2 PpIX induces apoptosis and activates TAp73 in p53-null cancer cells. a PpIX induces caspases as shown by the increase in the fluorescent signal in p53-null HCT 116 p53-/- and H1299 cancer cells. b PARP-1 cleavage in H1299 cells is induced by PpIX after $48 \mathrm{~h}$ treatment. c mRNA levels of NOXA and PUMA in H1299 cells treated with 1 mg/ml PpIX are elevated after 12 h. d, e PpIX induces TAp73 and its proapoptotic targets in H1299 (d) and HCT 116 TP53-/- (e) cancer cells. $20 \mu \mathrm{M}$ CDDP and $10 \mu \mathrm{M}$ Nutlin were used as positive controls for induction of p73. The uncropped blots for (d) are presented in Additional file 4: Figure S4a. f PpIX downregulates $\triangle$ Np73 protein levels in HCT 116 p53-/- cancer cells

In addition, the knockdown of TAp73 protected from induction of PARP-1 after treatment of H1299 cells with cisplatin (Additional file 2: Figure S2c). Thus, our data demonstrated that PpIX-activated TAp73 compensates for $\mathrm{p} 53$ loss and induces cancer cells' death in the absence of p53. 


\section{a}

$\mathrm{H} 1299$

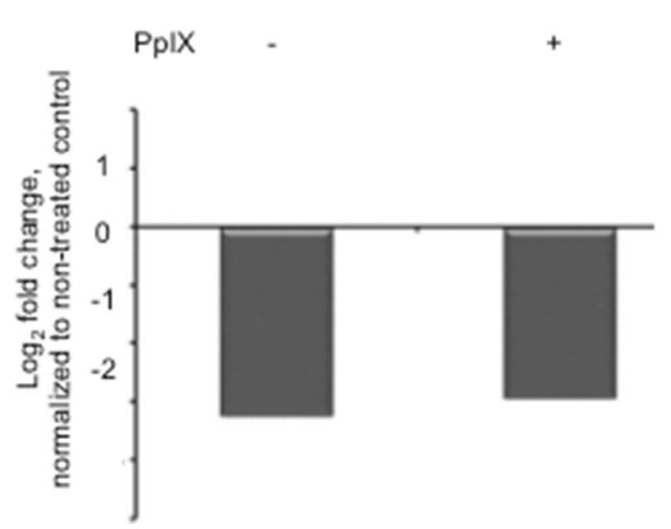

c

H1299

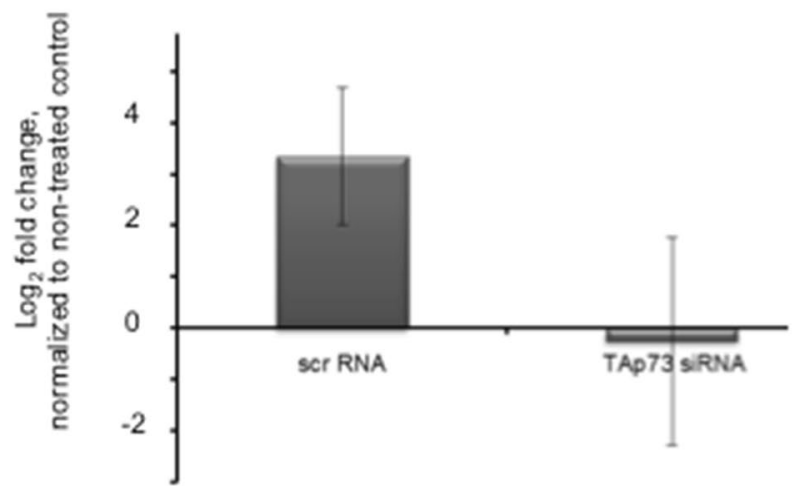

b $\mathrm{H} 1299$

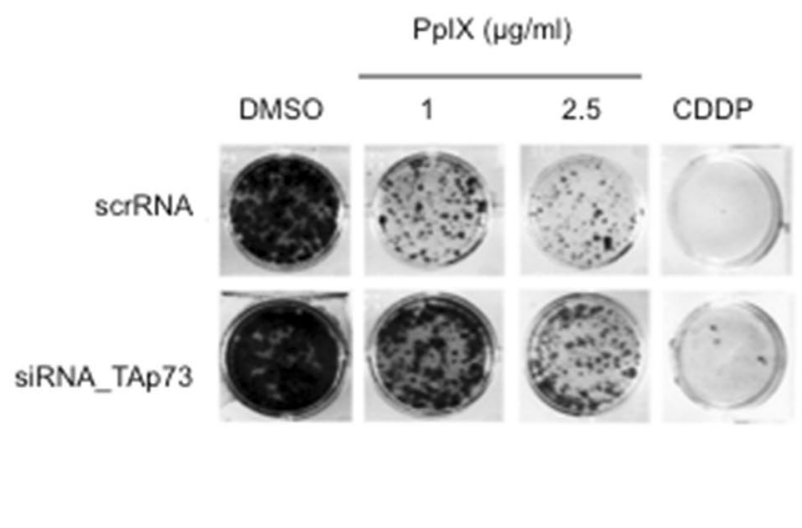

d

H1299

Fig. 3 TAp73 knockdown protects cancer cells from PpIX-induced cell death. a TAp73 knockdown with siRNA in H1299 cells as confirmed by qPCR. mRNA levels of TAp73 are not affected by $2.5 \mu \mathrm{g} / \mathrm{ml}$ PpIX. b TAp73 knockdown protects from PplX-induced growth inhibition in H1299 cells. c, $\mathbf{d}$ Ablation of TAp73 protects from induction of proapoptotic target Noxa by PpIX on mRNA (c) and protein levels (d)

Protoporphyrin IX activates TAp73 through the disruption of TAp73/MDM2 and TAp73/MDMX complex and protein stabilization

Our results showed that PpIX induces TAp73 transcription activity. In cancer cells, p73 transcription activity is abrogated, among other mechanisms, by binding to MDM2 or MDMX (human MDM4) [16]. We have shown previously that PpIX inhibits p53/MDM2 complex by binding to the N-terminal domain of p53 [14]. Since PpIX also binds to p73 [15] we addressed the question if PpIX abrogates interactions between TAp73 and MDM2 by using a defined yeast-based reporter system [17]. Briefly, we expressed TAp73 alone or together with MDM2 or MDM4 in a yeast strain harboring a chromosomally integrated luciferase reporter containing the p53 response element derived from the PUMA promoter. Our data manifested that PpIX restored the p73-dependent reporter in yeast strains expressing TAp73/MDM2 or p73/MDM4. This indicated that PpIX ablated TAp73/ MDM2 and TAp73/MDM4 interactions and promoted TAp73 transactivation function (Fig. 4a). To investigate if PpIX can inhibit TAp73/MDM2(X) interactions also in cancer cells, we immunoprecipitated TAp73 after treatment with PpIX and blotted the membrane with MDM2 or MDMX antibodies. Western blot showed inhibition of TAp73/MDM2 and p73/MDMX interactions by PpIX in H1299 and HCT 116 p53-/- cells (Fig. 4b, c). These findings are unexpected, since dual inhibitors of TAp73/ MDM2 and TAp73/MDMX interactions have not been described to date.

Since TAp73 protein levels (but not mRNA levels) were upregulated by PpIX in cancer cells (Fig. 2d, e), we performed pulse-chase experiments with cycloheximide (CHX) to assess whether PpIX increases the half-life of 


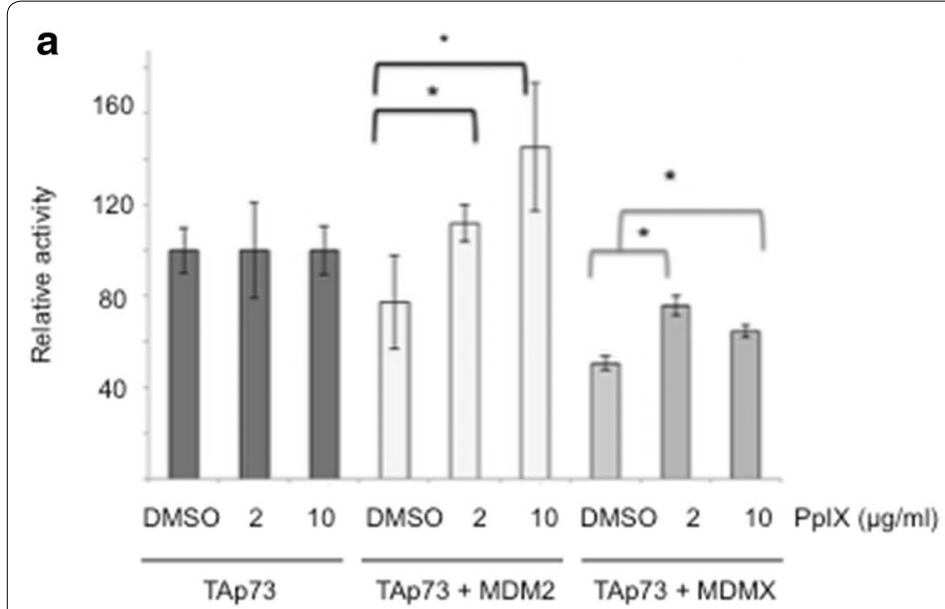

C

HCT 116 p53-\%

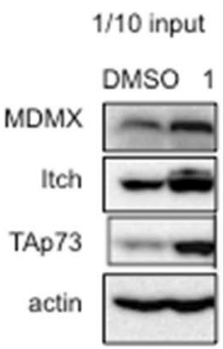

IP: TAp73

$\operatorname{lgG}$ DMSO $1 \mathrm{PplX}(\mu \mathrm{g} / \mathrm{ml})$

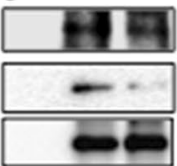

e

H1299

IP: TAp73

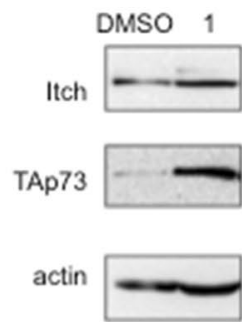

Fig. 4 PplX ablates TAp73/MDM2, TAp73/MDMX and TAp73/Itch complexes. a PplX rescues transcription activity of TAp73 thorough ablation of TAp73/MDM2 and TAp73/MDM4 interactions as assessed by yeast-based reporter system. The $t$-student test was performed for statistical analysis with $p \leq 0.05$. * Samples are considered statistically significant. b, c Disruption of TAp73/MDM2 (b) and TAp73/MDMX and TAp73/Itch (c) binding by PpIX is shown in co-immunoprecipitation experiment in HCT 116 p53-/- cells. Uncropped blots are presented in Additional file 5: Figure S5a and S5b. N Nutlin. $\mathbf{d}$ Chase experiment demonstrates stabilization of TAp73 by $1 \mu \mathrm{g} / \mathrm{ml} \mathrm{PpIX} \mathrm{in} \mathrm{H1299.} \mathrm{NT} \mathrm{non} \mathrm{treated} \mathrm{control.} \mathrm{e} \mathrm{TAp73/Itch} \mathrm{interaction} \mathrm{is}$ inhibited by PplX in H1299 by 1 mg/ml PplX. The uncropped blots are shown in Additional file 6: Figure S6a

TAp73 in cancer cells. We observed that PpIX decreased the TAp73 cellular turnover (Fig. 4d).

The key E3 ubiquitin ligase responsible for TAp73 degradation is Itch [7]. Small molecules inhibiting Itch b

HCT 116 p53-\%

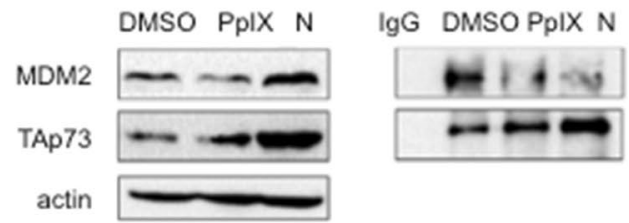

d

H1299

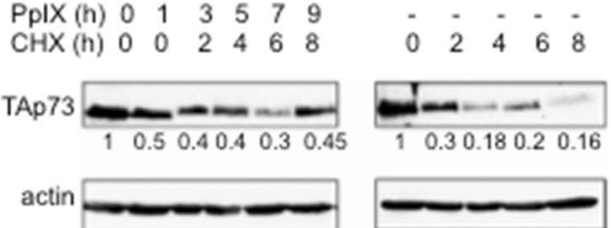

activity in cancer cells have recently been considered to have anticancer potential [18]. We reasoned that PpIX might inhibit TAp73/Itch interactions to induce TAp73 stabilization. We then co-immunoprecipitated TAp73 


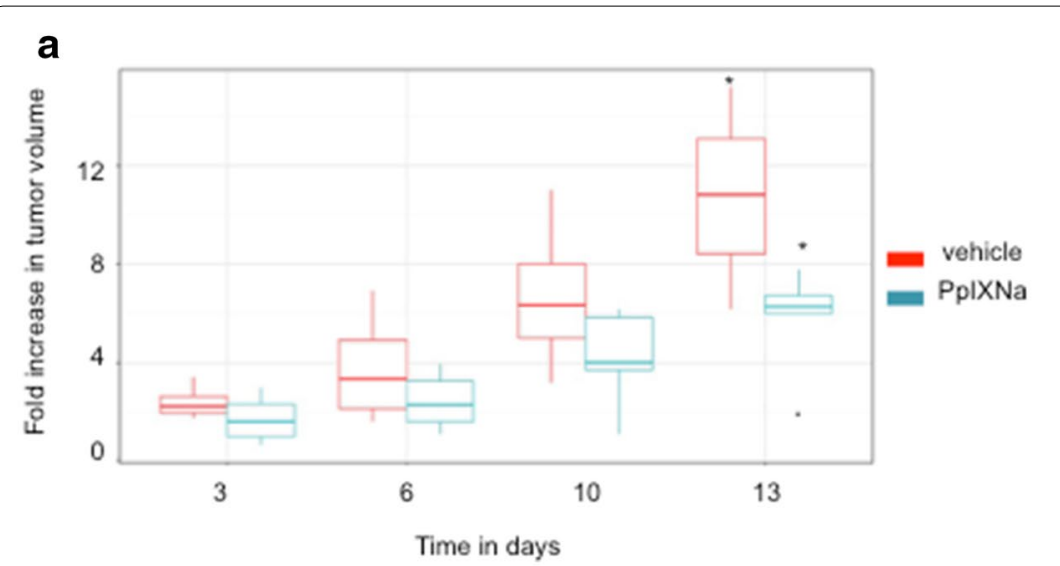

b

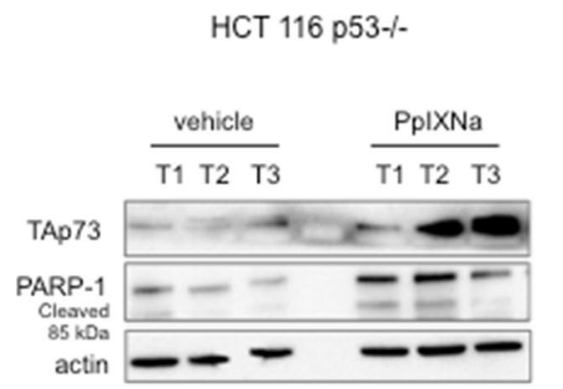

Fig. 5 Protoporphyrin IX inhibits the growth of HCT 116 p53-/ - xenograft tumors. a Inhibition of tumor growth after intravenous injections of PpIXNa (7.5 mg/kg/day) for day 1, 3, 6, 10 and 13. The results are shown as mean of SD ( $n=5$ for each group). $\mathbf{b}$ TAp73 is upregulated in PpIXNa-treated tumors with concomitant induction of PARP-1 cleavage

and demonstrated that PpIX inhibits TAp73/Itch in both H1299 and HCT p53-/- cell lines and the inhibition was more pronounced in H1299 cells (Fig. 4c, e).

Taken together, our data showed that PpIX activates TAp73-dependent apoptosis at several levels, namely, by promoting its transcriptional activity and protecting from proteasomal degradation.

\section{Protoporphyrin IX inhibits the growth of human xenograft tumors in mice}

To assess if protoporphyrin IX will also affect the tumor growth in mice, we injected SCID mice bearing HCT 116 p53-/- xenografts with sodium salt of protoporphyrin IX (PpIXNa). As shown in Fig. 5a, treatment with PpIXNa inhibited tumor growth and induced TAp73 at the protein level (Fig. 5b). Induction of TAp73 correlates with accumulation of the cleaved PARP-1. No weight loss was observed for PpIXNa- or vehicle-injected groups.

\section{Discussion}

It has been demonstrated that TAp73 $\mathrm{KO}$ mice are prone to carcinogen-induced tumorigenesis and that around $35 \%$ of mice cohort develop lung adenocarcinomas [19]. Acute genetic ablation of $\Delta \mathrm{N}$ isoforms triggers rapid regression of thymic lymphomas developed in p53-null mice [20]. Thus, deletion of $\Delta N p 63$ or $\Delta N p 73$ can compensate for p53 tumor suppression in thymic lymphomas, and this occurs due to accumulation of TA isoforms of p63 and p73 and induction of apoptosis [21]. Therefore, TAp73, similarly to $\mathrm{p} 53$, can be considered a promising therapeutic target in tumors deficient or mutant for TP53 gene.

Cancer-related deaths are a major health problem encountered today [22]. Therefore, there is an urgent need for the development of effective oncology drugs for tailored treatment of patients suffering from the metastatic disease. The success of anti-cancer treatment relies on the strategy that allows inhibition of cancer drivers without the induction of apparent side effects. However, such strategies are still at the early stage of development.

The p53 reactivation suppresses established tumors in vivo [23], yet this strategy is not implemented therapeutically. APR-246/MQ, a compound, in Phase II clinical development (clinical trial ID: NCT03268382), that binds to cysteine residues in mutant p53 and reactivates its function [24] brings hope for the patients harboring somatic or germline TP53 gene mutations.

Li-Fraumeni Syndrome is a rare genetic syndrome associated with the lifetime risk of cancer development. It is an autosomal dominant disorder associated with germline mutations in TP53 gene. The pre-cancerous niche in cancer-free LFS patients is driven by TP53 mutations [25]. Therapeutic approaches overriding the effect of the niche might delay if not completely inhibit the otherwise heavy tumor predisposition in LFS.

Due to the above and given that approximately around 2/3 of all human cancers harbour TP53 gene mutations (https://p53.fr/), a development of a new approach to compensate for p53 loss, is of the outmost clinical relevance. Small molecules reactivating TAp73 are of a special interest. Few molecules directly or indirectly activating TAp73 in cancer cells have been described in the literature. Small molecule Nutlin3, a potent inhibitor of p53/MDM2 interactions was found to activate p73 and induce p73-mediated apoptosis by disrupting p73/HDM2 interactions in cancer cells lacking p53 [9]. Next, small molecule RETRA was described to specifically suppress mutant p53-bearing tumor cells in vitro and in mouse 
xenografts by disrupting mtp53/p73 complex [26]. The widely-used genotoxic drug cisplatin (CDDP) was also shown to induce TAp73-mediated apoptosis in ovarian cancer cells irrespective of p53 status. Recent study showed that TAp73 sensitizes p53-null colon cancer cells to bortezomib and TAp73 was shown to compensate for p53-loss in induction of apoptosis after drug treatment [27].

Our previous findings showed that protoporphyrin IX binds to p53 and activates p53-dependent and independent cell death in colon cancer cells [14]. Consistently, PpIX was shown to induce cancer cells' death in sarcoma cells [28]. In line with our data (Fig. 1e), it was demonstrated that PpIX does not induce DNA damage but induces chromatin condensation by triggering the translocation of factors such as AIF from mitochondria to the nucleus, where it binds to the DNA and provokes caspase-independent chromatin changes [28].

TAp73 bears high structural and functional homology with p53. This prompted us to study if PpIX activates TAp73 in tumors lacking functional p53. Here, we showed that PpIX inhibits the growth of cancer cells lacking p53 in a dose-dependent manner. Fluorescentbased caspase assay indicated that the growth suppression resulted from induction of apoptosis. This is in line with previous study in which we observed inhibition of proliferation and the accumulation of HCT TP53-/cells in the sub-G1 fraction of the cell cycle after PpIX exposure [14].

Recently, a detailed analysis of the oligomeric state of TAp73 alpha by Melino's and Dötsch's labs revealed a high structure homology between p53 and p73 tetramers [29]. The study thus implies that p73 can be pharmacologically restored in a way similar to p53. This is supported by our previous and current findings, which showed that PpIX binds to p73 and induces accumulation of TAp73 and its apoptotic targets in cancer cells in a fashion similar to p53. Using siRNA we determined that TAp73 is crucial for induction of apoptosis in cancer cells treated with PpIX. This supports the notion that pharmacologically restored TAp73 behaves similarly to activated wild-type p53 and that TAp73 can compensate for p53 loss in cancer cells lacking TP53 gene.

To gain better insight into the mechanism of activation of TAp73 in cancer cells by PpIX, we used a yeastbased reporter system previously developed to screen for activators of p53 [17]. PpIX restored TAp73-dependent reporter in the presence of MDM2 or MDM4, inhibitors of p73 transcription activity. Thus, we concluded that PpIX abrogates TAp73/MDM2 and TAp73/MDMX interactions which leads to the induction of its transcription activity. In agreement with the above, PpIX inhibited TAp73/MDM2 and TAp73/MDMX interactions in cancer cells. This finding is novel since small- molecule, dual inhibitors of MDM2 and MDMX have not been described yet. So far, only a stapled peptide therapeutic, ALRN-6924, inhibiting both MDM2 and MDMX [30] has been developed and is currently in Phase I clinical trial in wild-type p53 AML and MDS (clinical trial ID: NCT02909972). Of note, our findings have important clinical relevance since the ability to disrupt the interactions of TAp73 with both MDM2 and MDMX is a favorable therapeutic approach in tumors with MDMX amplification.

Since mRNA levels of p73 were not elevated by PpIX we reasoned, that the increase on the protein level must be related to the prolonged stability of TAp73 in cancer cells. Chase experiments confirmed that PpIX stabilizes TAp73 on protein level. The stabilization was a consequence of disruption of interactions between TAp73 and E3 ubiquitin ligase, Itch in cancer cells. Furthermore, PpIX showed potent antitumor activity and inhibited the growth of HCT 116 p53-/- xenografts.

\section{Conclusions}

Taken together, our data showed that PpIX, a metabolite of aminolevulinic acid, activates TAp73 by several mechanisms converging on activation of its transcription activity and protein stabilization leading to the transactivation of pro-apoptotic PUMA and NOXA. Next, pharmacologically activated TAp73 compensates for p53 loss and induces apoptosis in cancer cells.

The concentrations of PpIX applied herein are those used for Photofrin ${ }^{\circledR}$-PDT, a clinically approved photosensitizer and an analog of PpIX, in in vitro studies [31]. Next, the dose of Photofrin ${ }^{\circledR}, 2-2.5 \mathrm{mg} / \mathrm{kg}$ applied in the clinical practice is injected once during the PDT treatment. The dose of PpIX that we have applied in the mouse xenograft studies is higher, however, it is expected since no light is needed to achieve the therapeutic effect. Thus, our findings, might in future lead to successful repurposing of porphyrins into clinical application to treat tumors with TP53 gene mutations.

\section{Methods}

All experiments, including protocols, were performed in accordance with the guidelines and regulations of Karolinska Institute and the University of Gdansk.

\section{Cell culture and chemicals}

The p53-deficient human colon cancer cells HCT 116 TP53-/- [32] (a generous gift from Prof. Bert Vogelstein, Johns Hopkins Medicine, Baltimore, MD, USA), human non-small cell lung carcinoma cell line H1299 (ATCC) and osteosarcoma Saos2 (ATCC) cells were cultured at $37^{\circ} \mathrm{C}$ in a humidified incubator with $5 \% \mathrm{CO} 2$. All 
cell lines were maintained in Iscove's modified Dulbecco's medium (Invitrogene, Sweden) supplemented with $1 \mathrm{mM}$ sodium pyruvate and 10\% FBS (Gibco, Sweden).

Protoporphyrin IX was purchased from Sigma-Aldrich, Germany and dissolved in 100\% DMSO (Sigma-Aldrich, Germany). The final concentration of DMSO in cell medium was $0.5 \%$. Cisplatin (CDDP) (Sigma-Aldrich, Germany) was dissolved in $\mathrm{dH}_{2} \mathrm{O}$ and used at final concentration $20 \mu \mathrm{M}$. Nutlin (Calbiochem, Sweden) was dissolved in DMSO and used at final concentration $10 \mu \mathrm{M}$.

\section{DNA constructs and siRNA}

For ectopic expression of TAp73alfa, we used pcDNA3.1TAp73 $\alpha$ construct kindly provided by Prof. Matthias Dobbelstein, University Medical Center Göttingen, Göttingen, Germany. The construct has been sequenced to confirm the fidelity of the transgene sequence. Transfection was performed with Lipofectamine 2000 (Invitrogen, Sweden) for $24 \mathrm{~h}$ according to the manufacturer's protocol.

The following siRNAs were used: TAp73_318 sense: AGGGCAUGACUACAUCUGU; antisense: ACAGAU GUAGUCAUGCCCU and TAp73_223 sense: ACCAGA CAGCACCUACUUC; antisense: GAAGUAGGUGCU GUCUGGU [33]. Transfection with siRNA (10 nM) was performed with HiPerfect reagent (Qiagene, Germany) for $24 \mathrm{~h}$ (for WB analysis) or $48 \mathrm{~h}$ (for colony formation assay or qPCR analysis) following manufacturer's instruction.

\section{Growth assay}

For short-term proliferation assay, HCT 116 p53-/cells were seeded at $5^{\wedge} 10^{3} /$ well in 96 -well plate and transfected with either the empty vector or pcDNA3 containing TAP73 $\alpha$ sequence. After $24 \mathrm{~h}$ cells were treated with PpIX and allowed to grow for another $24 \mathrm{~h}$. Cells' viability was measured by WST-1 proliferation reagent (Roche, Switzerland).

For a long-term colony formation assay, 3000 cells/well were seeded into twelve-well plates and treated with indicated concentrations of PpIX. The medium was changed after $3 \mathrm{~h}$ and cells were allowed to grow for 7 days. The colonies were stained with crystal violet reagent according to standard protocol.

\section{Quantitative PCR}

$48 \mathrm{~h}$ after siRNA transfection cells were harvested, mRNA was isolated and reverse transcribed to cDNA according to the manufacturer's instructions (5 Prime, Hamburg, Germany; Invitrogen, Sweden). For qPCR, the following concentrations were used: $150 \mathrm{nM}$ primers; 10 ng cDNA; $7.5 \mu \mathrm{l} 2 \times$ master mix (Bio-Rad, Sweden); water to a total of $15 \mu \mathrm{l}$; Primers used: TAP73 forward:
GGGAATAATGAGGTGGTGGG and TAP73 reverse: AGATTGAACTGGGCCATGAC, NOXA (PMAIP1) forward AAGTGCAAGTAGCTGGAAG, reverse: TGTCTC CAAATCTCCTGAGT, PUMA forward: CTCAACGCA CAGTACGAG and reverse: GTCCCATGAGATTGT ACAG, GAPDH forward: TCATTTCCTGGTATGACA ACG and reverse: ATGTGGGCCATGAGGT.

\section{Yeast-based reporter system}

The TAp73-dependent yeast reporter strain yLFMPUMA containing the luciferase CDNA cloned at the $A D E 2$ locus and expressed under the control of PUMA promoter response element was transfected with pTSGp73, pLS-MDM2 (derived from pRB254, generously provided by Dr. R. Brachmann, University of California, Irvine, CA, USA) [17], or pLS-Ad-MDM4 and selected on double drop-out media for TRP1 and HIS3. Luciferase activity was measured $16 \mathrm{~h}$ after the shift to galactosecontaining media and the addition of PpIX (SigmaAldrich, Germany), or DMSO. Presented are average relative light units and the standard errors obtained from three independent experiments each containing four biological repeats. Treatment with PpIX did not affect the transactivation of the reporter by TAp73 alone.

\section{Co-immunoprecipitation and western blotting}

For TAp73/Itch and TAp73/MDMX co-IP cells were treated for $24 \mathrm{~h}$ with $1 \mu \mathrm{g} / \mathrm{ml}$ PpIX and $30 \mu \mathrm{M}$ MG-132 was added $3 \mathrm{~h}$ before cell harvest. For p73/MDM2 co-IP cells were treated with PpIX or Nutlin for $24 \mathrm{~h}$ (HCT p53-/- cells). Cells for both whole cell lysates and immunoprecipitates were solubilized in lysis buffer: $25 \mathrm{mM}$ Tris $\mathrm{HCl}, \mathrm{pH}$ 8.0, $150 \mathrm{mM} \mathrm{NaCl}$ and $1 \%$ Nonidet P-40 (0.5\% for co-IP). For co-IP, $1 \mathrm{mg}$ protein was immunoprecipitated with $1.5 \mu \mathrm{g}$ of $\alpha$-TAp73 rabbit polyclonal antibody (Bethyl Laboratories, TX, USA) or normal mouse IgG (Millipore, MA, USA). Immuno-complexes were absorbed onto $40 \mu \mathrm{l}$ of Dynabeads ${ }^{\circledR}$ Protein A (Invitrogen, Sweden) for $5 \mathrm{~h}$ at $4{ }^{\circ} \mathrm{C}$. The immunoprecipitates were washed with $1 \mathrm{ml}$ of lysis buffer. The antibodies used for detection were: anti-p73 monoclonal antibodies [20] (IMG 246, Imgenex, UK), anti-MDM2 (Santa Cruz, Germany), anti-Itch (Calbiochem, Sweden), anti-MDMX (Bethyl Laboratories, TX, USA).

Western Blot was performed according to the standard protocol. $100 \mu \mathrm{g}$ of total cell lysate was subjected to electrophoresis and the following antibodies were used to detect proteins: anti-TAp73 (Bethyl Laboratories, TX, USA), anti-Bax (Santa Cruz, Germany) anti-PUMA (Cell Signaling), anti-Noxa (Calbiochem, Sweden), 
anti-PARP1/2 (Santa Cruz, Germany), anti-actin (SigmaAldrich, Germany).

\section{Caspase activation assay}

Activation of caspases by PpIX was measured with FAM-FLICA $^{\mathrm{TM}}$ Poly Caspase Assay Kit (ImmunoChemistry Technologies, Germany) according to manufacturer's protocol. Caspase activation was monitored by the means of flow cytometry with BD FacsCalibur after $6 \mathrm{~h}$ treatment with PpIX.

\section{Protein stability assay}

H1299 cells were pre-treated with $1 \mu \mathrm{g} / \mathrm{ml}$ PpIX for $1 \mathrm{~h}$ and translation was inhibited by adding cycloheximide (Sigma Aldrich, Germany) to final concentration $30 \mu \mathrm{g} /$ $\mathrm{ml}$. Cells were harvested 2, 4, 6 and $8 \mathrm{~h}$ after addition of $\mathrm{CHX}$ and subjected to Western blot analysis.

\section{Comet assay}

HCT p53-/- cells were seeded into 24-well plates and treated with PpIX $(1 \mu \mathrm{g} / \mathrm{ml}$ and $5 \mu \mathrm{g} / \mathrm{ml}), \mathrm{H}_{2} \mathrm{O}_{2}(100 \mu \mathrm{M})$ was used as a positive control. Following treatment, cells were pelleted by centrifugation at $1500 \mathrm{rpm}$ and suspended in $60 \mu \mathrm{l}$ of PBS (pH 7.4). $10 \mu \mathrm{l}$ of cell suspension was mixed with $100 \mu \mathrm{l}$ of $1 \%$ low melting agarose (Prona, Reducta LM, Poland) and $75 \mu$ of this cell-agarose mixture was spread on microscopic slides pre-coated with $1 \%$ agarose. A third layer of $0.5 \%$ low melting agarose $(75 \mu \mathrm{l})$ was applied over the layer of agarose with the cell suspension. Slides were incubated for $1 \mathrm{~h}$ in a lysis solution (2.5 M NaCl, $100 \mathrm{mM}$ EDTA, $100 \mathrm{mM}$ Tris, 1\% Triton $\mathrm{X}-100, \mathrm{pH} 10)$. The microscopic slides were immersed in an alkaline buffer $(300 \mathrm{mM} \mathrm{NaOH}, 1 \mathrm{mM}$ disodium EDTA) for $30 \mathrm{~min}$ after which they were subjected to electrophoresis at $1 \mathrm{~V} / \mathrm{cm}$ for $15 \mathrm{~min}$. Subsequently slides were neutralized with a neutralization buffer $(0.4 \mathrm{M}$ Tris, $\mathrm{pH} 7.5$ ) for $15 \mathrm{~min}$ and stained with ethidium bromide $(20 \mu \mathrm{g} / \mathrm{ml})$. Cells were analyzed under a fluorescence microscope (Nikon PCM-2000) using Cometscore ${ }^{\circledR}$ software. Images of 20 cells from three slides were analyzed. Densities were measured for each image in two areas: the whole cellular DNA and the area containing only the comet head. Results are presented as tail moment, which is the percentage of DNA in the comet tail multiplied by the tail length.

\section{Animal experiments}

Animal handling and housing were performed according to the regulations of Karolinska Institutet. All ethical approvals have been obtained (N334/12 and N635/12). SCID 6-week-old female mice were injected s.c. with
$5 \times 10^{6}$ HCT 116 p53-/- cells in PBS. Sodium salt of protoporphyrin IX (Sigma-Aldrich, Germany) was prepared as $100 \mu \mathrm{l}$ injections in PBS supplemented with 10\% DMSO. Intravenous injections were performed on day 1 , 3, 6, 10 and 13 with $7.5 \mathrm{mg} / \mathrm{kg}$ dose of PpIXNa. Vehicle was PBS supplemented with $10 \%$ DMSO.

\section{Statistical analysis}

Yeast assay was performed in 3 independent experiments with three triplicates. $\mathrm{p}<0.05$ was considered statistically relevant. The statistical significance was assessed by a parametric Student's t test (for variances Fisher-Snedecor's test was applied and the normality was estimated with the Shapiro-Wilk's test).

Tumor volume was analyzed according to the following description. In order to assess the significance of drug mixed model analysis of repeated measures data was performed.

The value of Chi square statistics, degrees of freedom for the statistic and corresponding $p$ value are: Chiseq 3.53; Chi df 1; Pr 0.06. One-sided Wald test was next performed: $\mathrm{t}$-statistics -2.228 and $\mathrm{p}$-value 0.01293 . Thus, PpIXNa treatment significantly reduced the tumor volume.

\section{Additional files}

Additional file 1: Figure S1. (a) PpIX induces caspases as shown as the increase in the fluorescent signal in p53-null Saos2 cells.

Additional file 2: Figure S2. (a) TAp73 depletion protects from PpIXinduced caspase activation in p53-null Saos2 cells. (b) TAp73 knockdown ablates PpIX-induced accumulation of PUMA and PARP-1 cleavage in H1299 cells. (c) Silencing of TAp73 partially inhibits PARP-1 cleavage in H1299 after CDDP treatment. Dotted line indicates sites where membrane was cut. The uncut blots are shown in (d).

Additional file 3: Figure S3. Uncropped versions of the blots presented in Fig. $1 \mathrm{~b}$ and $\mathrm{f}$, respectively. X-sample or compound not included in this study.

Additional file 4: Figure S4. Uncropped versions of the blots presented in Fig. 2d.

Additional file 5: Figure S5. Uncropped versions of the blots presented in Fig. 4b, C.

Additional file 6: Figure S6. Uncropped versions of the blots presented in Fig. 4e.

\section{Abbreviations}

TA: transcriptionally active; PpIX: protoporphyrin IX; CDDP: cisplatin; CXH: cycloheximide.

\section{Authors' contributions}

$A S, A K, A K, P A, M L$, prepared figures and drafted the manuscript; JZP and Al, designed the study, wrote and revised the manuscript. All authors read and approved the final version of the manuscript. 


\begin{abstract}
Author details
1 Department of Biotechnology, Intercollegiate Faculty of Biotechnology, University of Gdansk and Medical University of Gdansk, Abrahama 58, 80-307 Gdansk, Poland. ${ }^{2}$ Department of Microbiology, Tumor and Cell Biology, Karolinska Institutet, Biomedicum, Solnavägen 9, 17165 Stockholm, Sweden. ${ }^{3}$ Centre for Integrative Biology, CIBIO, University of Trento, via Sommarive 9, 38123 Trento, Italy. ${ }^{4}$ Present Address: Department of Molecular Biology, Massachusetts General Hospital, Harvard Medical School, Boston, MA 02114, USA.
\end{abstract}

\section{Acknowledgements}

We thank Prof. Galina Selivanova and Dr. Margareta Wilhelm for scientific discussions. Special thanks are addressed to Dr. Marcela Franco and Katarzyna Koczergo for a technical support, and to dr Alicja Szabelska for statistical analysis of data obtained from mouse xenografts experiment. We are grateful to Kenth Andersson and the animal facility Karolinska Institutet for excellent help. We also thank all our colleagues for sharing their reagents.

\section{Competing interests}

The authors declare that they have no competing interests.

\section{Consent for publication}

Not applicable.

\section{Data availability}

All data generated or analyzed during this study are included in the article [and its additional files].

\section{Ethics approval and consent to participate}

Not applicable.

\section{Funding}

Research was funded by the grant from the Polish National Science Center no 6126/B/P01/2010/38 NN405612638, Karolinska Institute and Stockholms Läns Landsting, Åke Wibergs stiftelse, IARC IG grant (\#12869), the Strategic Research Programe in Cancer (StratCan) and ETIUDA grant. JZP would like to acknowledge the award for Young Talented Scientist from Polish Ministry of Science and Higher Education.

\section{Publisher's Note}

Springer Nature remains neutral with regard to jurisdictional claims in published maps and institutional affiliations.

Received: 17 August 2018 Accepted: 9 November 2018

Published online: 26 December 2018

\section{References}

1. Pantziarka P, Pirmohamed M, Mirza N. New uses for old drugs. BMJ. 2018;361:k2701.

2. Dotsch V, Bernassola F, Coutandin D, Candi E, Melino G. p63 and p73, the ancestors of p53. Cold Spring Harbor Perspect Biol. 2010;2(9):a004887.

3. Tomasini R, MakTW, Melino G. The impact of p53 and p73 on aneuploidy and cancer. Trends Cell Biol. 2008;18(5):244-52.

4. Irwin MS, Kondo K, Marin MC, Cheng LS, Hahn WC, Kaelin WG Jr. Chemosensitivity linked to p73 function. Cancer Cell. 2003;3(4):403-10.

5. Han S, Semba S, Abe T, Makino N, Furukawa T, Fukushige S, Takahashi H, Sakurada A, Sato M, Shiiba K, et al. Infrequent somatic mutations of the p73 gene in various human cancers. Eur J Surg Oncol. 1999;25(2):194-8.

6. Wu H, Leng RP. MDM2 mediates p73 ubiquitination: a new molecular mechanism for suppression of p73 function. Oncotarget. 2015. https:// doi.org/10.18632/oncotarget.4086.

7. Rossi M, De Laurenzi V, Munarriz E, Green DR, Liu YC, Vousden KH, Cesareni G, Melino G. The ubiquitin-protein ligase Itch regulates p73 stability. EMBO J. 2005;24(4):836-48

8. Zhang P, Liu SS, Ngan HY.TAp73-mediated the activation of c-Jun $\mathrm{N}$-terminal kinase enhances cellular chemosensitivity to cisplatin in ovarian cancer cells. PLOS ONE. 2012;7(8):e42985.
9. Lau LM, Nugent JK, Zhao X, Irwin MS. HDM2 antagonist Nutlin-3 disrupts p73-HDM2 binding and enhances p73 function. Oncogene. 2008;27(7):997-1003.

10. Kostecka A, Sznarkowska A, Meller K, Acedo P, Shi Y, Mohammad Sakil HA, Kawiak A, Lion M, Krolicka A, Wilhelm M, et al. JNK-NQO1 axis drives TAp73-mediated tumor suppression upon oxidative and proteasomal stress. Cell Death Dis. 2014;5:e1484.

11. Agostinis P, Berg K, Cengel KA, Foster TH, Girotti AW, Gollnick SO, Hahn SM, Hamblin MR, Juzeniene A, Kessel D, et al. Photodynamic therapy of cancer: an update. CA Cancer J Clin. 2011;61(4):250-81.

12. Kaneko T, Tominaga M, Kouzaki R, Hanyu A, Ueshima K, Yamada H, Suga M, Yamashita T, Okimoto T, Uto Y. Radiosensitizing effect of 5-aminolevulinic acid and protoporphyrin IX on carbon-ion beam irradiation. Anticancer Res. 2018;38(7):4313-7.

13. Bednarz N, Zawacka-Pankau J, Kowalska A. Protoporphyrin IX induces apoptosis in HeLa cells prior to photodynamic treatment. Pharmacol Rep PR. 2007;59(4):474-9.

14. Zawacka-Pankau J, Issaeva N, Hossain S, Pramanik A, Selivanova G, Podhajska AJ. Protoporphyrin IX interacts with wild-type p53 protein in vitro and induces cell death of human colon cancer cells in a p53-dependent and -independent manner. J Biol Chem. 2007;282(4):2466-72.

15. Sznarkowska A, Malenczyk K, Kadzinski L, Bielawski KP, Banecki B, Zawacka-Pankau J. Targeting of p53 and its homolog $\mathrm{p} 73$ by protoporphyrin IX. FEBS Lett. 2011;585(1):255-60.

16. Wang X, Arooz T, Siu WY, Chiu CH, Lau A, Yamashita K, Poon RY. MDM2 and MDMX can interact differently with ARF and members of the p53 family. FEBS Lett. 2001;490(3):202-8.

17. Andreotti V, Ciribilli Y, Monti P, Bisio A, Lion M, Jordan J, Fronza G, Menichini P, Resnick MA, Inga A. p53 transactivation and the impact of mutations, cofactors and small molecules using a simplified yeast-based screening system. PLoS ONE. 2011;6(6):e20643.

18. Rossi M, Rotblat B, Ansell K, Amelio I, Caraglia M, Misso G, Bernassola F, Cavasotto CN, Knight RA, Ciechanover A, et al. High throughput screening for inhibitors of the HECT ubiquitin E3 ligase ITCH identifies antidepressant drugs as regulators of autophagy. Cell Death Dis. 2014;5:e1203.

19. Tomasini R, Tsuchihara K, Wilhelm M, Fujitani M, Rufini A, Cheung CC, Khan F, Itie-Youten A, Wakeham A, Tsao MS, et al. TAp73 knockout shows genomic instability with infertility and tumor suppressor functions. Genes Dev. 2008;22(19):2677-91.

20. Venkatanarayan A, Raulji P, Norton W, Chakravarti D, Coarfa C, Su X, Sandur SK, Ramirez MS, Lee J, Kingsley CV, et al. IAPP-driven metabolic reprogramming induces regression of $\mathrm{p} 53$-deficient tumours in vivo. Nature. 2015;517(7536):626-30.

21. Venkatanarayan A, Raulji P, Norton W, Flores ER. Novel therapeutic interventions for p53-altered tumors through manipulation of its family members, p63 and p73. Cell Cycle. 2015. https://doi.org/10.1080/15384 101.2015.1121333.

22. Global Burden of Disease Cancer C, Fitzmaurice C, Akinyemiju TF, Al Lami $\mathrm{FH}$, Alam T, Alizadeh-Navaei R, Allen C, Alsharif U, Alvis-Guzman N, Amini E, et al. Global, regional, and national cancer incidence, mortality, years of life lost, years lived with disability, and disability-adjusted life-years for 29 cancer groups, 1990 to 2016: a systematic analysis for the global burden of disease study. JAMA Oncol. 2018. https://doi.org/10.1001/jamao ncol.2018.2706

23. Ventura A, Kirsch DG, McLaughlin ME, Tuveson DA, Grimm J, Lintault L, Newman J, Reczek EE, Weissleder R, Jacks T. Restoration of p53 function leads to tumour regression in vivo. Nature. 2007;445(7128):661-5.

24. Zhang Q, Bykov VJN, Wiman KG, Zawacka-Pankau J. APR-246 reactivates mutant p53 by targeting cysteines 124 and 277. Cell Death Dis. 2018;9(5):439.

25. Pantziarka P. Primed for cancer: Li Fraumeni Syndrome and the precancerous niche. Ecancermedicalscience. 2015:9:541.

26. Kravchenko JE, Ilyinskaya GV, Komarov PG, Agapova LS, Kochetkov DV, Strom E, Frolova El, Kovriga I, Gudkov AV, Feinstein E, et al. Small-molecule RETRA suppresses mutant p53-bearing cancer cells through a p73-dependent salvage pathway. Proc Natl Acad Sci USA. 2008;105(17):6302-7

27. Dabiri Y, Kalman S, Gurth CM, Kim JY, Mayer V, Cheng X. The essential role of TAp73 in bortezomib-induced apoptosis in p53-deficient colorectal cancer cells. Sci Rep. 2017;7(1):5423. 
28. Li Q, Wang X, Zhang K, Li X, Liu Q, Wang P. DNA damage and cell cycle arrest induced by protoporphyrin IX in sarcoma 180 cells. Cell Physiol Biochem Int J Exp Cell Physiol Biochem Pharmacol. 2013;32(3):778-88.

29. Luh LM, Kehrloesser S, Deutsch GB, Gebel J, Coutandin D, Schafer B, Agostini M, Melino G, Dotsch V. Analysis of the oligomeric state and transactivation potential of TAp73alpha. Cell Death Differ. 2013;20(8):1008-16.

30. Carvajal LA, Neriah DB, Senecal A, Benard L, Thiruthuvanathan V, Yatsenko T, Narayanagari SR, Wheat JC, Todorova TI, Mitchell K, et al. Dual inhibition of MDMX and MDM2 as a therapeutic strategy in leukemia. Sci Transl Med. 2018. https://doi.org/10.1126/scitranslmed.aao3003.

31. Liu K, Chen W, Lei S, Xiong L, Zhao H, Liang D, Lei Z, Zhou N, Yao H, Liang Y. Wild-type and mutant p53 differentially modulate miR-124/iASPP feedback following pohotodynamic therapy in human colon cancer cell line. Cell Death Dis. 2017;8(10):e3096.

32. Bunz F, Dutriaux A, Lengauer C, Waldman T, Zhou S, Brown JP, Sedivy $J M$, Kinzler KW, Vogelstein B. Requirement for p53 and p21 to sustain G2 arrest after DNA damage. Science. 1998;282(5393):1497-501.

33. Stantic M, Sakil HA, Zirath H, Fang T, Sanz G, Fernandez-Woodbridge A, Marin A, Susanto E, Mak TW, Arsenian Henriksson M, et al. TAp73 suppresses tumor angiogenesis through repression of proangiogenic cytokines and HIF-1alpha activity. Proc Natl Acad Sci USA. 2015;112(1):220-5.
Ready to submit your research? Choose BMC and benefit from:

- fast, convenient online submission

- thorough peer review by experienced researchers in your field

- rapid publication on acceptance

- support for research data, including large and complex data types

- gold Open Access which fosters wider collaboration and increased citations

- maximum visibility for your research: over 100M website views per year

At BMC, research is always in progress.

Learn more biomedcentral.com/submissions 